ISSN: 0212-5374

\title{
LA FORMACIÓN DEL DOCENTE EN Y PARA LA COMPLEJIDAD
}

\section{Teachers' training in and for complexity}

\author{
José María ASENSIO AGUILERA
}

Universidad Autónoma de Barcelona. Facultad de Ciencias de la Educación. Departamento de Pedagogía Sistemática y Social. E-G 08193 Bellaterra.

Barcelona.Correo-e: josepmaria.asensio@uab.es

Fecha de aceptación definitiva: marzo de 2001

BIBLID [(1130-3743) 12, 2000, 29-43]

\section{RESUMEN}

Educar resulta en nuestro tiempo una actividad extremadamente compleja que requiere maestros y profesores formados en la experiencia y el dominio de unos saberes que les permitan abordar con éxito esa complejidad. Al respecto se argumenta que los enfoques interdisciplinares y sistémico-complejos, así como los conocimientos que permiten comprender mejor nuestras actividades mentales, resultan esenciales para la formación del docente y, en especial, para el desarrollo de su tacto pedagógico. Éste, aunque dependiente de aspectos profundamente enraizados de la personalidad del educador, puede verse acrecentado a partir de una adecuada reflexión sobre la práctica, que incluya, sobre todo, los elementos afectivos, epistemológicos y éticos inherentes a la misma.

Descriptores: aprendizaje por ósmosis, intuición, tacto pedagógico, complejidad.

\section{SUMMARY}

To educate is an extremely complex activity in our times, and it requires teachers trained in the experience and expertise of the knowledge that will help them to deal with this complexity. To this respect it is important to show up that interdisciplinary and complex systemic approaches, and also the knowledge that allows a better 
understanding of our mental activities are essential in teachers' training, specially in which respects to his or her pedagogical tact. This, although it depends on deeply rooted it aspects of the personality of the educator, it can turn increased mainly from an appropriate reflection on the practice that includes the affective epistemologic an ethical elements to this practice.

Key words: learny by osmosis, intuition, pedagogical tact, complexity, cognitive autonomy.

\section{INTRODUCCIÓN: DEL APRENDER PARA VIVIR AL VIVIR PARA APRENDER}

Se suele decir que vivimos en unos tiempos carentes de pasado, de narrativas, de modelos. Y si se destaca esta circunstancia es porque se considera implícitamente, al margen de otras valoraciones, que no ha sido la mencionada nuestra forma habitual de conducirnos. En efecto, hasta el descubrimiento de la escritura, los seres humanos adultos venían a ser algo así como el libro viviente del que los más jóvenes aprendían cuanto convenía para subsistir. Y aquello que convenía merecía esta valoración después de haber estado sometido a prueba - la de su utilidad para la supervivencia- por las anteriores generaciones. El espacio y el tiempo compartidos, la vida en común, eran la escuela en la que los niños asimilaban los comportamientos, actitudes y creencias de sus mayores. Se trataba, sobre todo, de observar e imitar, de aprender para sobrevivir, de generar un conocimiento implícito, unas destrezas, que fueran efectivas para afrontar los avatares de la vida cotidiana. La educación no era otra cosa que el resultado de la convivencia en el grupo humano del que se formaba parte. Y, en lo esencial, eso siguió siendo así, únicamente, para una gran mayoría de personas hasta casi llegar a los albores del siglo $\mathrm{xx}^{1}$.

En nuestras sociedades, las cosas, tanto en los mencionados como en otros muchos aspectos, han cambiado sustancialmente. El caudal de saberes disponibles ha aumentado de manera extraordinaria al mismo tiempo que los medios para codificarlos y transmitirlos. Lo considerado útil para la vida ya no se muestra a los menores en los mismos espacios/tiempos donde, de manera participativa, se desarrollaban antaño las actividades comunes al grupo, sino en lugares específicos (escuelas, institutos, universidades, etc.) separados del hacer comunitario. La relación directa de lo aprendido con la satisfacción de unas necesidades básicas ha desaparecido y, con ella, buena parte de la motivación intrínseca por conocer. La educación, en su papel difusor de esos inabarcables saberes, ya no se dirige tampoco a unas minorías privilegiadas sino que se pretende para la totalidad de la ciudadanía. Hoy, en definitiva, es preciso realizar un gran esfuerzo educativo, en tiempo,

1. Aún a finales del siglo Xvin sólo un quince por ciento de la población en Alemania sabía leer y escribir.

(C) Ediciones Universidad de Salamanca

Teor. educ. 12, 2000, pp. 29-43 
medios y dedicación, para que las nuevas generaciones accedan al conocimiento y a las experiencias que les permitan valerse en ese mundo casi virtual en que les ha correspondido vivir.

En un abrir y cerrar de ojos, la Humanidad ha pasado así de estar representada por unos colectivos que aprendían, de manera prioritaria, aquello que precisaban para vivir, a estarlo por otros, los actuales, que, cuanto menos en el mundo tecnológicamente desarrollado, parecen vivir sólo para aprender. O, simplemente, para consumir informaciones. Como expresa Claxton (1999: 21), en nuestros días

"La gente tiene prisa por saber, por conocer las respuestas, por plantear y resolver. Queremos explicaciones con carácter de urgencia. Teorías de cualquier cosa, desde las crisis matrimoniales al origen del universo. Deseamos tener más datos, más información. Y queremos que lleguen más rápido, y que, con el menor esfuerzo mental posible, nos digan claramente qué debemos hacer".

Diríase que pese al deslumbrante dominio científico-tecnológico alcanzado, sentimos el futuro incierto y soportamos peor que nunca las sensaciones de inseguridad por leves que éstas sean. Las grandes preguntas permanecen sin respuesta y los saberes de que disponemos más que protegernos de nuestros miedos e ignorancias, parecen significar tan sólo la llave que abre las puertas a nuevas e inquietantes preguntas. Unas interrogaciones que intuimos nos sitúan, en algunos casos, al límite de nuestras capacidades intelectuales para darles cumplida respuesta. De esta manera, educar en las llamadas sociedades de la información resulta, paradójicamente, una tarea más ardua e incierta que en cualquier otro período de la historia de la Humanidad.

En este mundo necesitado de sentido y sometido a continuas e imprevisibles mudanzas, dos cosas permanecen sin embargo inalterables: la necesidad de ser educados para poder adaptarnos en el espacio y en el tiempo, y las aptitudes de nuestras mentes para adquirir los conocimientos que se precisan para ello. Disponemos, pues, de un "viejo" cerebro ${ }^{2}$ para asimilar una complejidad que nos desborda. Y precisamos de una "nueva" escuela que, además de los medios tecnológicos y didácticos necesarios, disponga de unos enseñantes formados en y para la complejidad, preparados científica y pedagógicamente para educar en unos tiempos que parecen haberle dado la espalda a lo más humano de nuestra humanidad: nuestra condición de seres abiertos a la sabiduría, que es algo distinto a la mera acumulación de saberes, al amor y la ética. Porque el desafio de la educación en nuestras sociedades no es tanto, a nuestro modo de ver, el que plantea la capacitación técnico-científica como el de la formación de personas autónomas, responsables y dialogantes además de dispuestas mentalmente para apropiarse de un conocimiento integrador y no dogmático.

2. El cerebro humano apenas ha variado desde la aparición del Homo sapiens sapiens hace unos cincuenta mil años. 


\section{TODOS EDUCAMOS, ALGUNOS SON ENSEÑANTES}

La educación es, en la actualidad, el lugar común en el que confluyen muchas de nuestras lamentaciones y esperanzas. Denunciar lo que se considera son desatinos en la formación de las nuevas generaciones y manifestar lo que correspondería hacer para remediarlos es algo a lo que muy pocos ciudadanos se resisten. Todos nos sentimos legitimados para opinar con razón - y a veces hasta por buenas razones - acerca de la educación, para argumentar que si padres, profesores o instituciones educativas fomentaran tales o cuales conocimientos y valores, entonces todo cambiaría a mejor. Lastimosamente, con frecuencia nos pasa desapercibido el hecho, no ya de que con nuestro comportamiento contribuimos muchas veces a alimentar esos mismos males que señalamos, sino y sobre todo, que le pedimos a la educación cosas que ésta no puede darnos: diagnósticos precisos, orientaciones inequívocas acerca de lo que procede en cada momento, previsión certera de los resultados, cuando no que ejerza un notorio predominio de sus influencias sobre las que determina el medio sociocultural en cada sujeto. Es decir, se solicita indebidamente a la educación lo que se espera con el empleo de las técnicas surgidas de las ciencias físico-naturales.

Por otra parte, cada vez se aprecia hoy en mayor medida una tendencia a delegar en terceros el logro de nuestras propias expectativas educativas, a concebir la escuela como la gran responsable de la formación de nuestra juventud. Para unos políticos excesivamente pendientes de sus problemas de imagen y de los que genera su propio discurso, para unos medios de comunicación seducidos por los porcentajes de audiencia y para unas familias saturadas de obligaciones, sin duda representa un importante alivio poder distraerse de algunas de sus responsabilidades educativas. Pero ello no deja de alimentar un tipo de autoengaño que no sólo redunda negativamente en la educación de nuestros hijos, sino también en la valoración de los enseñantes, muchas veces desbordados por la problemática que se ven obligados a afrontar en sus respectivos centros escolares. Así, no resulta infrecuente constatar que muchas de las críticas dirigidas a los docentes involucran a quienes las formulan, porque la tarea de educar compete al conjunto de la sociedad y no únicamente a unas determinadas personas o instituciones.

Ante lo que se entiende - la educación- como un quehacer abierto a todo tipo de opiniones, y un conocimiento —el pedagógico— de limitada fiabilidad aplicativa, no puede sorprender en demasía la escasa consideración social que merecen a nuestros contemporáneos quienes se dedican profesionalmente al ámbito educativo. Un dominio en el que las soluciones técnicas a los problemas que se plantean no resulta siempre posible, ni sus efectos inmediatos ni tampoco fácilmente contrastables. Justo lo opuesto de lo que en nuestros días se reclama para satisfacer las demandas de todo orden que se considera deben ser atendidas.

Sin embargo, no todas las reticencias hacia la Pedagogía proceden de las influencias del vigente paradigma tecnológico, ni carecen de razonable fundamento. Desde el propio campo de la educación se pone a veces el dedo en la llaga 
acerca del especial estatus que ostenta la formación del docente al señalar, como lo hace Max van Manen (1998: 16), que "aunque una persona aprenda todos los métodos curriculares y todas las técnicas de instrucción, puede resultar un mal profesor». Es decir, al referirse a dicha formación en términos inusualmente aplicables a otras actividades: la buena preparación teórica de un ingeniero, un arquitecto o, aunque en menor medida, de un médico "anuncian" unas equiparables competencias profesionales. Cierto que el contacto con la realidad acabará en todos los casos mejorando éstas y también poniendo en evidencia algunas carencias personales o técnicas. Pero creemos que no hasta el extremo de poner esa praxis al descubierto unas limitaciones que hicieran merecer valoraciones análogas a la antes formulada respecto al ejercicio de la actividad docente.

La reflexión que sigue va a ir dirigida a analizar lo que entendemos suscitan esos juicios de valor: preguntarnos por la cualidad profesionalizadora de la formación pedagógica que reciben nuestros enseñantes, y las causas del porqué puede ésta resultar irrelevante para la práctica educativa en algunas ocasiones. Es decir, intentar explicar a qué obedece el hecho, antes referido, de que unas brillantes calificaciones académicas no sean garantía razonablemente suficiente para el buen hacer profesional. $O$, si se prefiere, dar razones de la excelencia de algunos profesores que no han recibido una formación académica orientada a la docencia. Para abordar todo ello partiremos de dos tipos de presunciones que no tienen por qué excluirse mutuamente. La primera apunta a la idoneidad de los curricula que se imparten en las facultades de Ciencias de la Educación. Éstos podrían resultar, en efecto, inadecuados en algún sentido y proporcionar una capacitación teórica insuficiente para afrontar en muchos casos, por sus solos méritos, los problemas educativos. La segunda nos lleva a pensar que lo esencial de la relación pedagógica, aquello que determina sobre todo la valoración del alumno hacia su profesor, no depende tanto de la formación disciplinar que se imparte en esas facultades como de otros conocimientos vinculados a la praxis educativa. En nuestra opinión, ambas conjeturas merecen ser consideradas si lo que se pretende es comprender la naturaleza de dicha práctica y orientar las correspondientes modificaciones curriculares que sirvan para mejorar la formación de los educadores en general y de los enseñantes en particular.

\section{La insuficiencia PedAGógica de las CienCias de la EduCaCión}

La Pedagogía vive de prestado por cuanto se refiere a los contenidos que maneja para explorar lo que constituye su específico objeto de estudio: la educación. Ésta constituye, como se sabe, el atractor en el que confluyen todas las reflexiones pedagógicas a las que ciertas disciplinas, asimismo dispuestas a elucidar cuestiones educativas, aportan algunos aspectos de sus respectivos cuerpos teóricos. Son las llamadas Ciencias de la Educación. Un conjunto de saberes a partir de los cuales (aunque no únicamente) la Pedagogía pretende eelaborar criterios que 
rijan cursos de acción" (García Carrasco y García del Dujo, 1996: 33) para así influir en la realización de unos determinados proyectos educativos. Se advierte, pues, que el conocimiento pedagógico que sedimenta el futuro educador durante su período de formación procede, esencialmente, de la asimilación de esos saberes que proponen un amplio abanico de materias. Y nos preguntamos, en consecuencia, por las razones de que el dominio de esas disciplinas curriculares se revele insuficiente, cuando no aparentemente innecesario, para determinar la calidad pedagógica de las actuaciones educativas.

Si partimos de la base de que el conocimiento que precisa el docente para intervenir en esos proyectos educativos es, sobre todo, aquel que le permita orientar procesos de cambio, establecer relaciones, integrar modelos de actuación e interpretar lo más adecuadamente posible un fluir continuo de situaciones, consideramos que la formación teórica de los educadores podría verse mejorada de potenciarse en sus estudios: a) una mayor incidencia de los contenidos teóricos que permiten elaborar una abierta teoría del sujeto y de sus procesos mentales, y b) una visión de los problemas educativos que transcendiera el ámbito disciplinario y aproximara al docente al tipo de conocimiento integrado que demandada el tratamiento de éstos. La adopción de estas medidas curriculares no eliminaría ciertamente las limitaciones inherentes a la complejidad de las relaciones educativas, pero sí contribuiría a la comprensión de las situaciones en las que aquéllas se manifiestan. Entre estas limitaciones destacaríamos las derivadas de:

\section{1) La inadaptación intrínseca del currículum a los momentos pedagógicos}

En las intervenciones pedagógicas se entremezclan de manera inseparable conocimientos empíricos y experienciales, juicios de valor e interpretaciones de conductas que convierten en ilusoria la suficiencia pedagógica de cualquier tipo de desarrollo curricular por idóneo que éste fuera. Ahora bien, al margen de ese curricularmente inaccesible mosaico de representaciones mentales que intervienen en las acciones pedagógicas, los conocimientos aportados por las diferentes Ciencias de la Educación revelan su insuficiencia para prescribir aquello que procede, por la sencilla razón de que no se refieren a un individuo particular, sino a un sujeto genérico, a una abstracción que se entiende legalmente representativa de nuestra común condición humana y del colectivo al que ese sujeto pertenece. No es esa entidad abstracta y ahistórica, sin embargo, la que el educador encuentra en su camino. Por el contrario, éste se sitúa siempre ante individuos con nombres y apellidos que, además de compartir con sus congéneres una misma bumanidad, presentan unas necesidades educativas concretas intimamente relacionadas con su particular historia personal. Se puede, en definitiva,

"Sacar algunas leyes generales y creer que el comportamiento individual está determinado por ellas pero, cuando se trata de la conducta de una persona precisa, las 
leyes estadísticas ya no valen, el individuo recobra en cierto modo toda su libertad" (Wittezaele y García, 1994: 334).

Aunque que esto sea así no quiere significar, en modo alguno, que el conocimiento de esas entidades genéricas no sea útil para delimitar/justificar el campo de intervención pedagógica y para no ir dando "palos de ciego" tanto en el diagnóstico de las situaciones que se le plantean al educador, como en la búsqueda de soluciones para las mismas.

$\mathrm{Al}$ enseñante le interesa pues conocer, por ejemplo, qué factores intervienen en la motivación de los alumnos de unas determinadas edades. Pero, finalmente, lo que aquél debe de decidir, sin que ninguna disciplina venga en su auxilio, es cuál de las estrategias motivacionales posibles conviene pedagógicamente para los casos reales que se ve en la responsabilidad de afrontar. Sin duda esclarecer de qué tipo de conocimientos echa entonces mano el educador para decantarse por una u otra alternativa constituye uno de los aspectos más controvertidos de las decisiones pedagógicas. A nuestro entender, como matizaremos más adelante, sólo un determinado tipo de conocimientos teóricos, de significación más bien antropológica que técnica, adquieren una verdadera relevancia en esa toma de decisiones.

\section{2) La falta de una visión inter y transdisciplinar de los problemas educativos}

La formación teórica del educador debiera beber prioritariamente, en nuestra opinión, en las fuentes del diálogo inter y transdisciplinar, entendido éste como "una transformación recíproca de tales o cuales disciplinas en relación con este o aquel sujeto-objeto-contexto complejo" (Vilar, 1997: 29). Pero, lamentablemente, nos encontramos con otro tipo de realidad en los estudios que se imparten en las facultades de Ciencias de la Educación. En efecto, a pesar de que las materias que cursan los futuros docentes o pedagogos tratan, en la bella teoría, de aspectos relacionados con la educación, lo cierto es que los programas impartidos acaban olvidando, con excesiva frecuencia, esa anunciada vinculación de sus contenidos con las demandas de la Pedagogía. La conexión de ésta con las demás Ciencias de la Educación no llega así a producirse o lo hace de manera débil e indirecta. A través, en el mejor de los supuestos, de un encuentro pluridisciplinar que no facilita, con su mera yuxtaposición de conocimientos, la interpretación pedagógica de las interacciones educativas.

El estudio parcelado de realidades cuya comprensión no admite ese tratamiento analítico priva de una visión más profunda e integrada de aquéllas y, por consiguiente, de la posibilidad de encontrar soluciones eficaces para los problemas que generan. El enfoque sistémico-complejo, que interpreta la realidad en términos de "conectividad, relaciones y contexto" (Capra, 1998: 230), representa en este sentido un valioso instrumento transdisciplinar para concebir, modelizar y operar en cualquier tipo de situaciones que, como las educativas, tienen en la complejidad 
uno de sus rasgos distintivos. Aproximarse a la resolución creativa de los problemas complejos, como destaca Ferrer (1998: 230),

"precisa diferentes disciplinas y fundamentalmente, esencialmente, precisa una forma de pensar específica, el pensamiento sistémico, que integra aquellas disciplinas en un cuerpo coherente teórico-práctico, y les impide actuar como recursos separados".

Esta integración de conocimientos, por otra parte, no le resta protagonismo al desarrollo disciplinario del que depende, sino que lo estimula al generar nuevos modelos interpretativos y heurísticas que contribuyen a esclarecer la problemática que aborda cada materia desde sus propios planteamientos teóricos.

Antes, no obstante, de pasar a considerar algunos aspectos relacionados con el enfoque sistémico-complejo de interés para la practica educativa, abordaremos el segundo de los supuestos que, como se recordará, hacía referencia a la importancia de los saberes curriculares en la "reflexión" que solicitan las acciones pedagógicas.

\section{LA FORMACIÓN DEL EDUCADOR Y LOS MOMENTOS PEDAGÓGICOS}

La afirmación de Max van Manen, que entiendo suscribiría la mayor parte de los observadores de la realidad educativa, y el hecho, igualmente constatable, de que muchos profesores de reconocida calidad no hayan recibido formación pedagógica, nos puede llevar a pensar que, desde la perspectiva del educando, la valoración de las relaciones que establece con su profesor depende, esencialmente, de aspectos que quedan al margen de los saberes disciplinarios adquiridos por éste. Nos abre a la consideración, en suma, de que lo que se "cuece" en las instancias anteriores o posteriores a los momentos pedagógicos tiene menor significación respecto a dicha valoración que lo acontecido en el "cara a cara" profesor-alumno. $\mathrm{O}$ sea, que lo experimentado en un ámbito de decisión en que el tiempo (no ha lugar para dilaciones en la respuesta) y el espacio en que se desenvuelven los actores se comprimen, la memoria de trabajo no puede contextualizar debidamente el alud de técnicas de que dispone el enseñante y se hacen necesarios otro tipo de conocimientos, además de los académicos, para orientar el curso que han de seguir las interacciones pedagógicas.

Es cierto que algunos autores han señalado la circunstancia antes apuntada y la necesidad de modificar determinados aspectos de la formación de quienes deben tratar con realidades inabarcables desde unos conocimientos formales. Así, Schön (1992: 25) señala que:

"La cuestión de la relación entre la competencia en la práctica y el conocimiento profesional precisa ser planteada al revés. No deberíamos empezar por preguntar cómo hacer mejor uso del conocimiento científico sino qué podemos aprender a partir de un detenido examen del arte, es decir, de la competencia por la que en realidad los prácticos son capaces de manejar las zonas indeterminadas de la práctica 
independientemente de aquella otra competencia que se puede relacionar con la racionalidad técnica».

Desde la Pedagogía se ha reiterado, al respecto, la necesidad de formar profesionales "reflexivos" capaces de evaluar el desarrollo de las relaciones educativas y de justificar críticamente sus conjeturas y decisiones pedagógicas. Pero entendemos que, con frecuencia, se obvian aspectos de esa reflexión vinculada a la práctica que no debieran ser pasados por alto ya que hacerlo enmascara las diferentes estrategias mentales que se adoptan en la misma. Veamos.

Con independencia de si se realiza en los momentos previos a la acción pedagógica (qué pretendo, cómo planifico mis intervenciones, etc.) simultáneos a su desarrollo (cómo regulo mis influencias en función de lo que acontece) o posteriores (qué observo asociado a las actuaciones que llevo a cabo y cómo lo evaluo), al termino "reflexión" se le acostumbra a tomar en su sentido habitual de "pensar" o "razonar". De valorar, en suma, las ventajas y los inconvenientes de unas posibles acciones. En realidad pues, más que distinguir, como hace Elliot (1997), tres modalidades distintas de reflexión asociadas a la acción educativa, sería mejor hablar de una misma clase de actividad mental, la del pensamiento lógico, racional y consciente, aplicado a tres situaciones diferentes. La cuestión a resaltar de todo ello es la de que ni el pensamiento deliberativo es la única función mental conducente a la interpretación de determinadas situaciones o problemas, ni tampoco la forma habitual de "reflexión" en las relaciones "cara a cara" entre profesores y alumnos, educadores y educandos.

Como destaca Claxton (1999), existen, además del pensamiento reflexivo, otras modalidades de procesamiento mental que intervienen en nuestra valoración de las situaciones y la correspondiente toma de decisiones. Una de ellas, la emocional, actúa a mayor velocidad que el propio pensamiento. Nos proporciona una primera e inmediata evaluación de las cosas que nos afectan, orienta nuestras acciones y facilita la resolución de ciertos problemas, además de los procesos de memoria asociados a los acontecimientos vividos. La otra modalidad de "pensamiento" distinta a la reflexión consciente, viene asociada a los procesos intuitivos, es más lenta que ésta (aunque sus "productos" o intuiciones pueden emerger súbitamente a la conciencia) y resulta de elaboraciones mentales que precisan, por lo general, un cierto período de "incubación" de las ideas que nutren ese proceso mental preconsciente. Esta forma de "pensar" más relajada, que no busca tanto la resolución de unos problemas como la contemplación o exploración de los mismos, resultaría especialmente adecuada "cuando se trata de desvelar relaciones ocultas entre distintas áreas de conocimiento y de entender los patrones que conectan experiencias a parentemente dispares" (Claxton, 1999: 83). El pensamiento reflexivo no es, por consiguiente, el único instrumento mental del que puede disponer el enseñante para adecuar sus actuaciones, y el hecho de que tanto la "inteligencia" emocional como la intuición respondan a instancias menos conscientes del individuo no le resta importancia en su obrar. Como tampoco le priva de la posibilidad 
de poder conocer y manejar, en cierto grado, los respectivos patrones de emergencia de esas valoraciones no deliberadas.

La importancia de estas otras formas de evaluación mental se acrecienta en situaciones que, como muchas de las educativas, requieren una inmediata ponderación y respuesta. Es decir, mientras los momentos previos y posteriores a la acción pedagógica admiten reflexiones "de despacho", no constreñidas por el factor tiempo, y permiten disponer de todos los conocimientos que pueden traerse a mano, la "reflexión" en la acción adolece, por lo común, de esas posibilidades. Escapa, en gran medida, a la deliberación consciente, se nutre de aprendizajes por ósmosis que nos pasan desapercibidos, responde a sentimientos que se despiertan de manera imprevista y utiliza tanto conocimientos teóricos como extraídos de la experiencia del sujeto que han sedimentado en una especie de sabiduria o conocimiento tácito (Polanyi, 1967) de difícil transmisión.

La "reflexión en la acción" no es, en definitiva, una actividad mental semejante al pensamiento analítico, fácilmente verbalizable, ni se alimenta de los mismos procesos que las destrezas. Mediante el aprendizaje por ósmosis los sujetos llegan a advertir de manera inconsciente (aunque empíricamente contrastable) los elementos comunes que subyacen a una multitud de experiencias siempre distintas pero no absolutamente dispares. A través de esta modalidad de aprendizaje las personas pueden llegar a desvelar relaciones y patrones que el mero conocimiento teórico no permite hacer evidentes y que la reflexión tampoco acostumbra a desvelar en la conciencia de aquéllas. No se da, por consiguiente, una traslación directa de ese conocimiento teórico asimilado a la sabiduría que regula y dirige el sentido de las acciones en las situaciones educativas. Se puede disponer así, como apuntaba Max van Manen, de un brillante currículum académico y ser un "mal profesor" si éste carece de esa inteligencia práctica que se nutre de la experiencia y elabora la mente al margen de nuestra atención. "El tacto pedagógico — señala este autorno es una habilidad que podamos utilizar, es algo que somos" (Manen, 1998: 211).

La intuición entendida como un "mecanismo encubierto por el que llegamos a la solución de un problema sin razonar sobre él" (Damasio, 1996: 178) se muestra análogamente como un proceso mental, distinto al pensamiento reflexivo, que nos permite acceder (aunque según algunos estudios no a todos por igual) a nuevos significados y explicaciones, utilizando para ello como "materia prima" el conjunto de informaciones y conocimientos de los que ya disponemos. La intuición asoma, por lo general, cuando no se pretende deliberadamente alcanzar la comprensión de algo que se nos muestra indefinido, difícil de conceptualizar o de relacionar a partir de nuestros conocimientos teóricos y los adquiridos a través de la experiencia.

Al igual que el aprendizaje por ósmosis, los procesos intuitivos se revelan, en consecuencia, como formas "lentas" y no conscientemente pretendidas de "pensamiento" que desempeñan un papel destacado en las relaciones humanas y, por supuesto, en las educativas. O sea, en ámbitos donde los detalles, los pequeños cambios conductuales, las variaciones de actitud o las modificaciones en el entramado de relaciones que se detecta, adquieren una gran significación para decantarse por 
una u otra secuencia de acciones. Y donde, también, como es el caso del "cara a cara" profesor-alumno, cada uno de los componentes de esa díada, percibe cosas del otro que a éste le pasan inadvertidas. Somos, en consecuencia, para los demás, distintos de como creemos ser y ello induce muchas veces al enseñante a cometer errores de apreciación en las relaciones con sus alumnos.

Destacar que la "reflexión en la acción" no es una "verdadera" reflexión y que la experiencia resulta esencial para la formación del docente no pretende en cualquier caso sugerir que el pensamiento deliberado o la formación teórica del educador sea algo accesorio para su actividad profesional. Tanto lo detectado por ósmosis como aquello que la intuición ofrece a la conciencia han de ser evaluados por una racionalidad consciente sustentada por un cierto conjunto de supuestos críticamente establecidos. Lo contrario equivaldría a suponer, gratuitamente, que cuanto se elabora de manera menos consciente tiene mayores garantías de certeza que el conocimiento dependiente de la actividad reflexiva. Conviene tener presente, además, que la mente no trabaja "in vacuo", sino que elabora sus nuevas y, en ocasiones, fulgurantes conjeturas o intuiciones a partir de las ideas de que dispone. Se puede decir así que tanto más creativo se puede llegar a ser respecto a algo cuanto, dándonos el tiempo y las circunstancias para ello, mayor sea el bagaje de conocimientos relativos a ese algo del que dispongamos.

Como ya se ha comentado, consideramos, por otra parte, que los saberes que en mayor grado pueden contribuir a facilitar la "reflexión en la acción" y a superar las dificultades que plantean los momentos de intervención pedagógica, no son tanto los de tipo técnico como aquellos que presentan una especial significación desde el punto de vista epistemológico, antropológico y ético. Una significación que incide en los modelos mentales del educador así como en la transformación de éstos en bábitos de valoración y decisión que fluyan de manera espontánea en las relaciones pedagógicas. Son, en efecto, esos conocimientos los que creemos mejor pueden desarrollar en el docente los automatismos asociados a la sensibilidad perceptiva y valorativa que demandan las interacciones educativas, y que le van a permitir liberarlo de la necesidad de efectuar reflexiones fuera de lugar. Así, entendemos que quien deba proponerse de manera autocontrolada y consciente apreciar a sus alumnos, ser solicito con ellos, sentir la necesidad bumana y profesional de observar su evolución o de acogerlos en sus errores y dificultades, tiene escasas posibilidades de ser aceptado por ellos. Porque en educación, como en cualquier otro ámbito de las relaciones humanas, las técnicas de trato personal que no se armonizan e integran con el verdadero sentir del sujeto acaban siendo advertidas por los demás como elementos artificiales y manipulativos que, finalmente, provocan un más o menos disimulado rechazo hacia quien las exhibe. 


\section{EL PROYECTO DE EDUCAR}

Quienes se dedican a la tarea de educar advierten en ella, más pronto que tarde, junto a ciertas regularidades, un continuo reguero de particularidades y paradojas, de acontecimientos que se producen de manera uniforme junto a otros que lo hacen de forma imprevisible, de actuaciones efectivas para unos sujetos que no lo son para otros ni para esos mismos sujetos en distintas circunstancias. De influencias mínimas que ocasionan cambios notables allá donde otras, más persistentes, se han mostrado ineficaces o han llegado incluso a inducir el efecto opuesto al que se pretendía. Educar supone, en definitiva, orientarse entre el orden y la contingencia.

Los docentes saben, igualmente, que para desarrollar su labor mediadora no pueden confiarlo todo a la razón, a la lógica de sus argumentos, a la claridad de sus explicaciones. Los sentimientos están permanentemente presentes en las relaciones educativas. El enseñante sólo de manera artificial puede separar lo cognitivo de lo emocional, la aptitud intelectual de la actitud que el sujeto manifiesta respecto a cualquier objeto. En educación, el ser, el decir y el hacer no son pedagógicamente separables, como tampoco lo son, al enseñar y aprender, el conocer y el sentir.

Pese a la conciencia que indudablemente adquieren de lo inextricable del sistema con el que interactúan, maestros y profesores se sienten tentados muchas veces por la idea de creer que la técnica puede socorrerles en la mayor parte de sus problemas. Tienden a buscar la causa explicativa de cualquier acontecimiento complejo, a devorar métodos didácticos que les permitan "transmitir" sus conocimientos, a desconsiderar, por exigencias del programa o por convicción, la dimensión emocional o lúdica en sus relaciones educativas. Corren el riesgo así de olvidar que se dirigen a sujetos dotados de autonomía, capaces de realizar sutiles e inconscientes valoraciones afectivas y cognitivas de su entorno, sensibles a multitud de influencias y poseedores de historias que los particularizan en sus demandas educativas. Sistemas de estas características no resultan descifrables por el pensamiento analítico, ni manejables por técnicas sencillas, porque nada es la única causa de algo ni nadie el único responsable de ese algo.

El educador, el enseñante, se encuentra inmerso en la complejidad. Y, como ya se ha señalado, en ella y para ella entendemos que debe ser formado. Ofreciéndole, en definitiva, útiles mentales que le permitan hacer más inteligibles los problemas educativos, matizar el sentido de las acciones pedagógicas que lleva a cabo y establecer puentes de diálogo entre quienes tienden a destacar de la educación la relevancia de los datos o de su interpretación, lo genérico o lo particular, lo tecnológico o lo intuitivo, lo determinable o lo imprevisible.

\section{El aCCESO a la COMPLEJIDAD}

La idea de complejidad, aunque definida de múltiples maneras, se reconoce comúnmente asociada a cualquier cosa o sistema "compuesto de partes 
que interaccionan conjuntamente de forma heterogénea" y que "parece ser a la vez ordenada y desordenada, regular e irregular, variante e invariante, estable e inestable" (Edelman y Tononi, 2000: 163-164). Estos rasgos definitorios de lo complejo conllevan que lo así considerado no pueda ser descrito en su totalidad, ni tampoco precisado el sentido de sus posibles cambios. La complejidad escapa pues a nuestra plena comprensión y control, más no a poder ser interpretada y manejada en alguna medida a partir de ciertos principios interpretativos y de una visión abierta, relacional e integrada de los fenómenos que tienen al ser humano por observador. Es en este sentido que se puede considerar al pensamiento sistémico-complejo como "una forma de abordaje, un estilo cognitivo, un proyecto siempre vigente" (Droeven y Najmanovich, 1997: 42). Una manera, en definitiva, de contemplar la realidad sabiendo que, muchas veces, el estudio por separado de sus partes constitutivas no nos dice gran cosa acerca de los aspectos esenciales de la misma, ni de cómo sus cambios afectan al conjunto de elementos con los que se relaciona.

Los principios y teorías que contribuyen a comprender, explicar y gestionar la complejidad tienen procedencia diversa. Algunos de ellos emergen ya a principios del siglo pasado pero no es hasta la llegada de la llamada segunda Cibernética ${ }^{3}$, en la década de los setenta, cuando, de hecho, se abren las puertas a la consideración de todo aquello que resulta irreductible a la fragmentación. Con la aparición de la segunda Cibernética se plantea, en efecto, el papel de ese observador que nunca es exterior a la realidad observada, así como del lenguaje en la experiencia de conocer. Se destaca la autonomía del sujeto respecto al medio (también su dependencia de él), se advierte la convivencia simultánea de los procesos de cambio (estructurales) y de permanencia (de los patrones organizativos), así como la indeterminación de los itinerarios evolutivos que siguen los sistemas alejados del equilibrio (nosotros mismos, por ejemplo). El constructivismo se carga de razones, a partir de los avances de la biocognición, para alejarse drásticamente de la pretensión positivista de poder explicar la realidad con independencia de las operaciones mentales que realizan los sujetos y sus descripciones lingüísticas, para referirse a la experiencia de esa realidad. Sobre las representaciones mentales, destaca Damasio (1999: 318),

"no tengo ninguna idea del grado de fidelidad con que las imágenes mentales o las configuraciones neuronales se refieren a un objeto. Además, sea cual fuere el grado de fidelidad, las configuraciones neuronales y las configuraciones mentales correspondientes son al menos tan creaciones del cerebro como el producto de la realidad exterior que las suscita".

3. La segunda Cibernética o Cibernética de segundo orden (la primera, como se sabe, arranca de la obra de N. Wiener Cibernetics en 1948 y de las reuniones promovidas por la Fundación Macy en New York) fue impulsada, entre otros autores, por H. von Foerster, H. A. Simon, G. Bateson, E. Glasersfeld, H. Maturana y F. Varela. 
La aparición de la ciencia del caos y el descubrimiento de cómo evolucionan las estructuras alejadas del equilibrio, a las que Prigogine (1996) denomina "disipativas", representan, asimismo, un marco de referencia transdisciplinar, una "metáfora cultural" (Briggs y Peat, 1999: 8) para interpretar la complejidad. La dinámica de cambios que experimentan dichas estructuras nos habla de la imposibilidad de predecir la evolución de cualquier sistema (la mente, la sociedad, etc.) que tenga capacidades autoorganizativas ${ }^{4}$ al ser éste perturbado. Además nos indican que pequeñas influencias pueden ser suficientes para desencadenar transformaciones - crisis - susceptibles de producir cambios radicales o bifurcaciones en el sistema que los experimenta.

Los supuestos anteriores son de una gran relevancia para la Pedagogía. Presentan ese calado epistemológico, antropológico y ético al que me refería al tratar de la formación del educador. E incorporan una dimensión de lo humano que si bien no propone directamente una u otra técnica de actuación, impregna de sentido todas las decisiones del docente. Dichos supuestos, en efecto, proporcionan una visión constructivista del conocimiento (no existe un mundo independientemente de cómo es conocido/descrito) y advierten de la producción autónoma de sentido para cada sujeto. El lenguaje no es ese ideal vehículo transmisor que imaginara el positivismo lógico. Cuando las palabras encierran una elevada carga teórica, describen fenómenos de los que claramente participa el observador o se emplean respecto a objetos de conocimiento ampliamente interconectados, se aprecia cómo los vocablos, aun siendo los mismos, no representan ya idéntica cosa para cada sujeto. El sentido de lo expresado en la intención del emisor ya no aflora, en definitiva, con igual facilidad en quien le escucha como cuando el lenguaje se refiere a ámbitos de la realidad que, a efectos prácticos, admiten descripciones unívocas.

El enseñante deja de ser el epicentro en los procesos de enseñanza-aprendizaje. No está en sus manos especificar los cambios internos que conducen a la aparición de los aprendizajes, ni determinar la evolución que pretende para el educando. Pero no por ello desmerece su papel facilitador en el desarrollo personal y cognitivo de éste, al extremo de que sus influencias pedagógicas, por leves que éstas puedan parecer, pueden constituirse muchas veces en elementos desencadenantes de esas perturbaciones o crisis que afectan directamente a dicho desarrollo.

En este marco conceptual la verdad sin acotaciones desaparece como pretexto para justificar las acciones de los seres humanos. Los principios sistémico-complejos nos abren a una realidad susceptible de ser interpretada a partir de diferentes lógicas, conocida sólo a través de las operaciones mentales que realizan los sujetos y valorada únicamente en términos relativos. Se afirma así un sentido de la ética basado en la responsabilidad, el respeto a los demás y el diálogo como elemento imprescindible en las relaciones humanas, no tanto por su valor para consensuar

4. La autoorganización representa la capacidad de los sistemas de autogenerar nuevas estructuras y comportamientos al sufrir alguna perturbación. 
pareceres como para comprender el significado que quienes dialogan otorgan a la realidad que describen. Como señala de Tuca (1997: 49):

"... la respuesta explícita o implícita que cada uno de nosotros da a la cuestión de la realidad es la que determina cómo la persona vive su vida, igual que determina su aceptación o rechazo de otros seres humanos en la red de los sistemas sociales que la persona integra".

El valor pedagógico de los principios a los que se ha hecho referencia para el acceso a la complejidad, digámoslo una vez más, no sólo debe ser considerado en su vertiente didáctica, en el qué debo de hacer para enseñar sino, sobre todo, en el campo más genérico y trascendente de cómo se conciben al sujeto y a las relaciones educativas. Porque no en vano se educa teniendo siempre in mente una determinada imagen del ser humano conformadora, al igual que conformada, de ciertas creencias actitudes y valores.

\section{BiBLIOGRAFÍA}

Briggs, J. P. y PeAT, F. D. (1999): Las siete leyes del caos. Grijalvo, Barcelona.

CAPRA, F. (1998): La trama de la vida. Anagrama, Barcelona.

Claxton, G. (1999): Cerebro de liebre, Mente de tortuga. Urano, Barcelona.

DAmasio, A. R. (1996): El error de Descartes. Grijalbo, Barcelona.

- (1999): Le sentiment même de soi, corps, émotions, conscience. Odile Jacob, París.

Droeven, J. M. y Najmanovich, D. (1997): "De la cibernética a la complejidad: el devenir de la reflexión". Más allá de pactos y traiciones. Paidós, Buenos Aires.

Edelman, G. E. y Tonino, G. (2000): Comment la matière devient conscience. Odile Jacob, París.

Elirot, J. (1990): La investigación-acción en educación. Morata, Madrid.

García Carrasco, J. y García del Dujo, A. (1996): "Epistemología Pedagógica II". Teoría de la Educación, Revista Interuniversitaria, 8, 5-42.

FERRER, L. (1998): Del paradigma mecanicista de la ciencia al paradigma sistémico. Universitat de València, Valencia.

Manen, M. van (1998): El tacto en la enseñanza. Paidós, Barcelona.

Polanyt, M. (1967): The Tacit Dimension. Doubleday, Nueva York.

Prigogine, I. (1996): La fin des certitudes. Odile Jacob, París.

SCHÖN, D. A. (1992): La formación de profesionales reflexivos. Paidós, Barcelona.

VILAR, S. (1997): La nueva racionalidad. Kairós, Barcelona.

Wittezaele, J. L. y García,T. (1994): La escuela de Palo Alto. Herder, Barcelona. 